Proc. VII National Conference of Neutron Scattering and Complementary Methods, Chlewiska, Poland, June 12-16, 2011

\title{
Magnetic and Neutron Diffraction Studies of the Polycrystalline and Nanoparticle $\mathrm{TbMnO}_{3}$
}

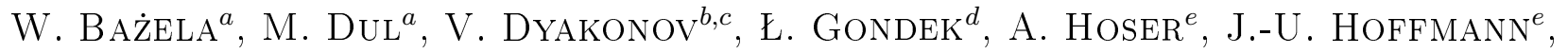 \\ B. Penc $^{f}$, A. Szytula ${ }^{f, *}$, Z. Kravchenko ${ }^{c}$, I. Nosalev $^{c}$ And A. Zarzycki ${ }^{g}$ \\ ${ }^{a}$ Institute of Physics, Technical University of Cracow, Podchorążych 1, 30-084 Kraków, Poland \\ ${ }^{b}$ Institute of Physics, PAS, al. Lotników 32/46, 02-668 Warszawa, Poland \\ ${ }^{c}$ A.A. Gałkin Donetsk Physico-Technical Institute, NANU, R. Luxemburg 72, 83114 Donetsk, Ukraine \\ ${ }^{d}$ Faculty of Physics and Applied Computer Science, AGH University of Science and Technology \\ al. A. Mickiewicza 30, 30-059 Kraków, Poland \\ ${ }^{e}$ Helmholtz-Zentrum Berlin für Materialen and Energie GmbH, Hahn-Meitner-Platz 1, 14-109 Berlin, Germany \\ ${ }^{f}$ M. Smoluchowski Institute of Physics, Jagiellonian University, W.S. Reymonta 4, 30-059 Kraków, Poland \\ ${ }^{g} \mathrm{H}$. Niewodniczański Institute of Nuclear Physics, PAS, E. Radzikowskiego 152, 31-342 Kraków, Poland
}

This paper reports on investigations of magnetic properties, crystal and magnetic structures on $\mathrm{TbMnO}_{3}$ prepared in various ways, namely, as conventional polycrystalline sample and two nano particle specimens (synthesized with a sol-gel method at temperatures of 800 and $850^{\circ} \mathrm{C}$ ). The X-ray and neutron diffraction data confirm the orthorhombic crystal structure (space group Pbnm, No. 62) without noticeable differences of the lattice parameters for poly- and nanocompounds. For the polycrystalline sample, a subsequent ordering of the Mn and Tb sublattices with decreasing temperature was observed. Namely, the Mn sublattice exhibits a modulated magnetic structure with the propagation vector $\boldsymbol{k}=\left(0, k_{x}, 0\right)$ in between $41-5 \mathrm{~K}$. Below $T=21 \mathrm{~K}$, a change from a collinear ( $A_{y}$ mode) into non-collinear $\left(A_{y} G_{z}\right.$ mode) structure was evidenced. Further decreasing of temperature below $10 \mathrm{~K}$ results in magnetic ordering of the $\mathrm{Tb}$ sublattice (modulated $G_{x} A_{y} F_{z}$ mode). For nanoparticle compounds, magnetic ordering in the $\mathrm{Mn}$ and $\mathrm{Tb}$ sublattices is described by propagation vector $\boldsymbol{k}=\left(0, k_{y}, 0\right)$, with $k_{y}$ components higher than observed for polycrystalline sample. The magnetic ordering in the Mn sublattice is described by a collinear $A_{y}$ mode down to $1.6 \mathrm{~K}$ where the $\mathrm{Tb}$ moment becomes ordered $\left(G_{x} A_{y}\right.$ mode). The observed broadening of the Bragg peaks connected to the Tb sublattice suggests the cluster-like character of its magnetic structure.

PACS: 75.40.Cx, 75.47.Lx, 75.25.-j, 75.50.Ee

\section{Introduction}

$\mathrm{RMnO}_{3}$ manganites, where $\mathrm{R}$ is the rare-earth element, have been recently a subject of intensive studies. The interest of researchers was aimed at their intriguing physical properties such as colossal magnetoresistivity, ferroelastic, and complex magnetic properties.

At room temperature, $\mathrm{TbMnO}_{3}$ compound exhibits the orthorhombically distorted perovskite structure (space group Pbnm). Magnetic susceptibility of the powdered sample obeys the Curie-Weiss law down to low temperatures with the small anomaly near $40 \mathrm{~K}$ and broad maximum at about $7 \mathrm{~K} \mathrm{[1].} \mathrm{Below} 40 \mathrm{~K}$, neutron diffraction (ND) data indicate a modulated magnetic structure of the Mn sublattice described by the propagation vector $\boldsymbol{k}_{\mathrm{Mn}}=(0,0.28,0)$. The Mn moments were found to form a collinear structure of $A$-type along the $b$-axis. Below $21 \mathrm{~K}$, the transition into a noncollinear structure described by the $A$ - and $G$-type modes was evidenced. Below $T=7 \mathrm{~K}$, the additional peaks connected with magnetic ordering of the Tb-sublattice with

* corresponding author; e-mail: andrzej.szytula@uj.edu.pl propagation vector $\boldsymbol{k}_{\mathrm{Tb}}=(0,0.415,0)$ were observed. Analysis of the patterns yields the complex structure (existence of satellites of $A, G$ and $C$-types) and short-range character of this ordering [1].

The new neutron diffraction data $[2,3]$ for the single crystal indicate the complex magnetic structure. Below $T_{\mathrm{t}}=28 \mathrm{~K}$, the additional weak satellite reflections of $C$ and $F$-types and third connected with the Mn sublattice were observed. The existence of the third harmonics was also reported in paper [4]. The new ND data for polycrystalline sample presented by Blasco et al. [5] give the model of the magnetic ordering similar to the previous model reported by Quezel et al. [1].

The low temperature ND data for the Tb-sublattice indicate the existence of the additional peaks with the propagation vector $2 k_{y}$ [2]. The results obtained in papers [3, 4] confirm the model presented in Ref. [1]. Detailed investigations of magnetic and electric properties related to the phase transitions were reported in Refs. $[6,7]$, in which the $(T, H)$ magnetic phase diagram was also presented. It should be mentioned that below $28 \mathrm{~K}$ the electric polarization connected with the occurrence of ferroelectric phase was observed [6]. 
It is well established that physical properties of manganites are heavily dependent on doping as well as on various intrinsic inhomogeneities, among which the nanostructure deserves the special attention. Due to nanosize crystallites, the nanoparticle compounds exhibit properties, which are different from those observed for the bulk materials. An important factor is increasing surface-to-volume ratio of the grains as the particle size is reduced to the nanoscale. As a result, the size effects and the surface effects perturb the properties of nanoparticle samples. In such samples, the magnetic behavior is additionally modified by the inter-particle interactions, which can be both of the exchange and magnetostatic origin.

In this paper, we discuss an influence of grain's size induced effects on the magnetic properties as well as on the crystal and magnetic structures of $\mathrm{TbMnO}_{3}$. Therefore, detailed investigations in terms of magnetic and neutron diffraction have been carried out in order to answer to the above addressed issue. Our data collected for two nanosized specimens as well as a reference conventional powder sample (micro-sized) give apparent evidence of above mentioned influence of size-effects on magnetic properties of $\mathrm{TbMnO}_{3}$.

\section{Experimental details}

The preparation of the $\mathrm{TbMnO}_{3}$ nanospecimens is described in Ref. [8]. The nanospecimens obtained by annealing at 800 and $850^{\circ} \mathrm{C}$ possess the average crystallite size of 45 and $60 \mathrm{~nm}$, respectively, and in the manuscript will be referenced as nano- 800 and nano- 850 . The grain size was estimated with both the BrunauerEmmett-Teller (BET) method and X-ray diffraction measurements [8]. Analysis of the powder X-ray diffraction data suggests that all the samples have the orthorhombic crystal structure described by the Pbnm space group. The dc magnetic measurements were carried out using a commercial MPMS SQUID magnetometer in the magnetic fields up to $50 \mathrm{kOe}$ in the temperature range $2-300 \mathrm{~K}$. Powder diffraction patterns were collected using the $E 2$ for polycrystalline sample and $E 6$ for nanosamples diffractometers installed at the BERII reactor (Helmholtz-Zentrum Berlin) within the temperature range from 1.6 to $260 \mathrm{~K}$. The incident neutron wavelengths were equal to $2.40 \AA$ for $E 2$ and $2.447 \AA$ for $E 6$. The neutron diffraction data were analyzed using the Rietveld-type program FullProf [9].

\section{Results}

\subsection{Crystal structure}

The parameters of crystal structure of the studied samples were determined by neutron diffraction experiment performed in their paramagnetic state $(51 \mathrm{~K}$ for polycrystalline sample and $50 \mathrm{~K}$ for nanosized specimens). Figures 1 and 2 present neutron diffraction patterns of $\mathrm{TbMnO}_{3}$ for polycrystalline and nano- 800 samples measured at different temperatures. The patterns at 51 and
$50 \mathrm{~K}$ were indexed within the orthorhombic structure of Pbnm space group. Tb and O1 atoms occupy two 4(c) sites $(x, y, 1 / 4)$; Mn atoms - 4(b) site $(1 / 2,0,0)$ and O2 atoms $-8(\mathrm{~d})$ site $(x, y, z)$. The presence of minor impurity phases was detected (see peak for $2 \theta=40.4^{\circ}$ on the diagrams in Fig. 2). The above findings were confirmed by X-ray diffraction data. The obtained results for reference sample have confirmed that its structure is stable at 5-260 K.

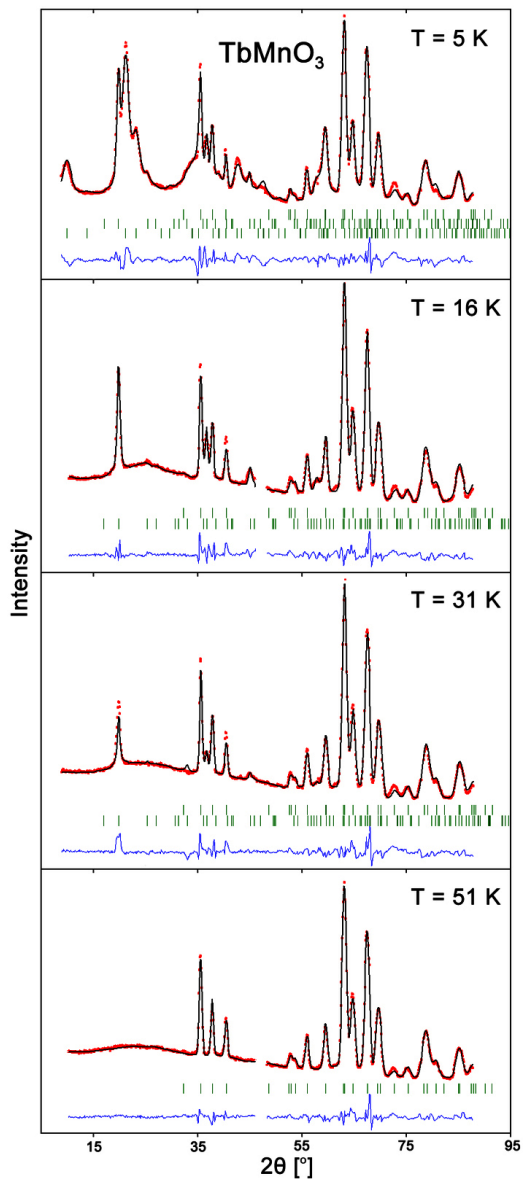

Fig. 1. Neutron diffraction patterns of polycrystalline $\mathrm{TbMnO}_{3}$ at $T=5,16,31$ and $51 \mathrm{~K}$. The symbols represent the experimental data while the solid line denotes the calculated profile. The difference between the observed and calculated intensities is shown at the bottom of each diagram. The vertical bars indicate the positions of the Bragg peaks, first row nuclear, second and third magnetic connected with the Mn- and Tb-sublattices.

The lattice constants and atomic positional parameters of $\mathrm{Tb}, \mathrm{O} 1$ and $\mathrm{O} 2$ atoms refined at 51 and $50 \mathrm{~K}$ are gathered in Table I. The parameters for polycrystalline sample are in good agreement with the data obtained by Blasco et al. (see Table 1 in Ref. [5]). The reliability factors are less than $5 \%$ yielding good accuracy of the performed analysis. Any significant difference in the structural parameters of poly- and nanosamples was no found except $x_{\mathrm{Tb}}$ parameters. 


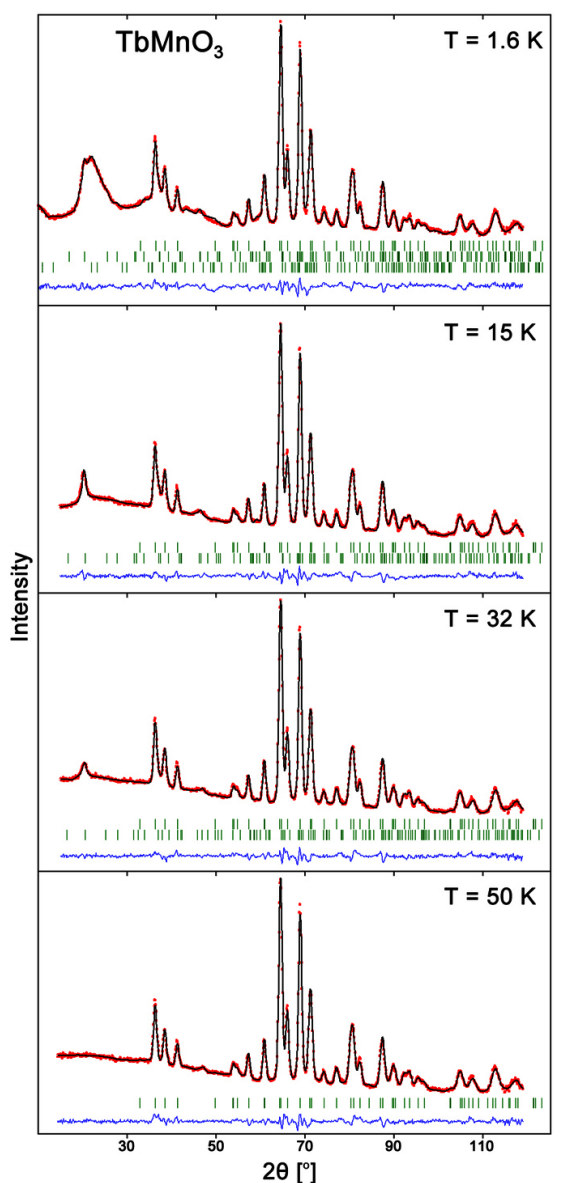

Fig. 2. Neutron diffraction pattern of nano-800 sample measured at 1.6, 15, 32 and $50 \mathrm{~K}$. Description similar as in Fig. 1.

\section{TABLE I}

Crystal structure parameters of polycrystalline and nanosized $\mathrm{TbMnO}_{3}$ compounds refined on the basis of neutron diffraction patterns collected at 51 and $50 \mathrm{~K}$. Standard deviations are given in parentheses. The atoms occupy following positions: $\mathrm{Tb}$ at $4 \mathrm{c}\left(x_{\mathrm{Tb}}, y_{\mathrm{Tb}}, 1 / 4\right)$; Mn at $4 \mathrm{~b}(1 / 2,0,0)$; $\mathrm{O} 1$ at $4 \mathrm{c}\left(x_{01}, y_{01}, 1 / 4\right)$ and $\mathrm{O} 2$ at $8 \mathrm{~d}\left(x_{02}, y_{02}, z_{02}\right)$.

\begin{tabular}{c|c|c|c}
\hline \hline Parameters & Polycrystalline & Nano-800 & Nano-850 \\
\hline$a[\AA]$ & $5.3170(11)$ & $5.3209(7)$ & $5.3257(7)$ \\
$b[\AA]$ & $5.8348(13)$ & $5.8205(7)$ & $5.8237(6)$ \\
$c[\AA]$ & $7.4202(20)$ & $7.4351(10)$ & $7.4454(11)$ \\
$V\left[\AA^{3}\right]$ & $230.20(16)$ & $230.27(9)$ & $230.92(9)$ \\
$x_{\mathrm{Tb}}$ & $0.9973(13)$ & $0.9843(7)$ & $0.9837(7)$ \\
$y_{\mathrm{Tb}}$ & $0.0772(10)$ & $0.0785(5)$ & $0.0794(5)$ \\
$x_{01}$ & $0.1108(14)$ & $0.1061(7)$ & $0.1056(7)$ \\
$y_{01}$ & $0.4685(10)$ & $0.4687(5)$ & $0.4699(6)$ \\
$x_{02}$ & $0.7086(11)$ & $0.7047(6)$ & $0.7038(6)$ \\
$y_{02}$ & $0.3272(8)$ & $0.3235(5)$ & $0.3229(5)$ \\
$z_{02}$ & $0.0504(6)$ & $0.0523(4)$ & $0.0520(4)$ \\
$R_{\text {Bragg }}[\%]$ & 4.93 & 3.75 & 4.66 \\
$R_{\text {prof. }}[\%]$ & 4.91 & 2.70 & 3.48
\end{tabular}

Table II shows the $\mathrm{Mn}-\mathrm{O}$ distances and $\mathrm{Mn}-\mathrm{O}-\mathrm{Mn}$ bond angles obtained from the refined atomic positions together with the $\mathrm{MnO}_{6}$ octahedron distortion defined as $D_{\text {oct }}=10 \times \sum\left(\mid(\mathrm{Mn}-\mathrm{O})_{i}-\right.$ $\left.\langle\mathrm{Mn}-\mathrm{O}\rangle_{\text {average }} \mid\right) /\langle\mathrm{Mn}-\mathrm{O}\rangle_{\text {average for each sample. The }}$ data for polycrystalline sample are in good agreement with those reported in Ref. [5]. It is worth noting that for nanosamples the octahedron's distortion is lowered in comparison to reference sample.

TABLE II

Interatomic $\mathrm{Mn}-\mathrm{O}$ distances $[\AA]$, octahedron distortion $D_{\text {oct }}$ and angles (deg) within the $\mathrm{MnO}_{6}$ octahedral for $\mathrm{TbMnO}_{3}$ poly- and nanosamples.

\begin{tabular}{c|c|c|c}
\hline \hline No. & Poly & Nano- $800{ }^{\circ} \mathrm{C}$ & Nano- $850{ }^{\circ} \mathrm{C}$ \\
\hline $\mathrm{Mn}-\mathrm{O}_{1}: 2$ & 1.955 & 1.951 & 1.952 \\
$\mathrm{Mn}-\mathrm{O}_{2}: 2$ & 2.239 & 2.210 & 2.205 \\
2 & 1.886 & 1.917 & 1.924 \\
$\langle\mathrm{Mn}-\mathrm{O}\rangle_{\text {average }}$ & 2.027 & 2.026 & 2.027 \\
$D_{\text {oct }}$ & 2.10 & 1.82 & 1.76 \\
$\mathrm{Mn}-\mathrm{O}_{2}-\mathrm{Mn}: 4$ & 146.24 & 145.78 & 145.61 \\
$\mathrm{Mn}-\mathrm{O}_{1}-\mathrm{Mn}: 2$ & 143.93 & 144.66 & 144.88 \\
$\langle\mathrm{Mn}-\mathrm{O}-\mathrm{Mn}\rangle_{\text {av }}$ & 145.47 & 145.41 & 145.37
\end{tabular}

\subsection{Magnetic properties}

Magnetic susceptibilities as a function of temperature are shown in Fig. 3. Figure 3a presents this dependence at low temperatures. The maximum at $9 \mathrm{~K}$ for polycrystalline sample and at $6.7 \mathrm{~K}$ for both nanosamples are observed. The maxima for nanosamples have the broad character. The increase of magnetic susceptibilities is observed below 4.4 and $4.0 \mathrm{~K}$ for poly- and nanosamples, respectively. In the temperature range $50-300 \mathrm{~K}$ the reciprocal magnetic susceptibilities obey the CurieWeiss law with the negative values of the paramagnetic Curie temperature equal to $-26.5 \mathrm{~K}$ for polycrystal and -29 and $-30 \mathrm{~K}$ for nanoparticle samples. The effective magnetic moment for polycrystalline sample equal to $10.98 \mu_{\mathrm{B}}$ is in agreement with value calculated from relation $\mu_{\mathrm{eff}}=\sqrt{\left(\mu_{\mathrm{eff}}^{\mathrm{Tb}}\right)^{2}+\left(\mu_{\mathrm{eff}}^{\mathrm{Mn}}\right)^{2}}$ for the free ions and equal to $10.88 \mu_{\mathrm{B}}$. For nanosamples the $\mu_{\mathrm{eff}}$ values are equal to $10.7 \mu_{\mathrm{B}}$ and $10.6 \mu_{\mathrm{B}}$ for nano- $850^{\circ} \mathrm{C}$ and nano$800^{\circ} \mathrm{C}$, respectively. The evidenced lowering of the effective magnetic moment resemble behavior of other nano sized perovskites. Namely, for $\mathrm{LaMnO}_{3+\delta}$ this was attributed to enhancement of the $\mathrm{Mn}^{4+}$ contribution [10].

Isothermal magnetization curves in magnetic fields up to $50 \mathrm{kOe}$ are shown in Fig. 4. The character of $M(H)$ isotherms for all the samples at $2 \mathrm{~K}$ indicate the metamagnetic character of magnetization with the critical field determined from the field dependence of $\frac{\mathrm{d} \mu}{\mathrm{d} H}$, equal to $H_{\mathrm{cr}}=17.4 \mathrm{kOe}$. The metamagnetic character of the magnetization curves was observed only in low temperatures, thus it may be attributed to the Tb magnetic sublattice. As apparent from Fig. 4, the magnetization does not achieve saturation in magnetic field up to $50 \mathrm{kOe}$ and 

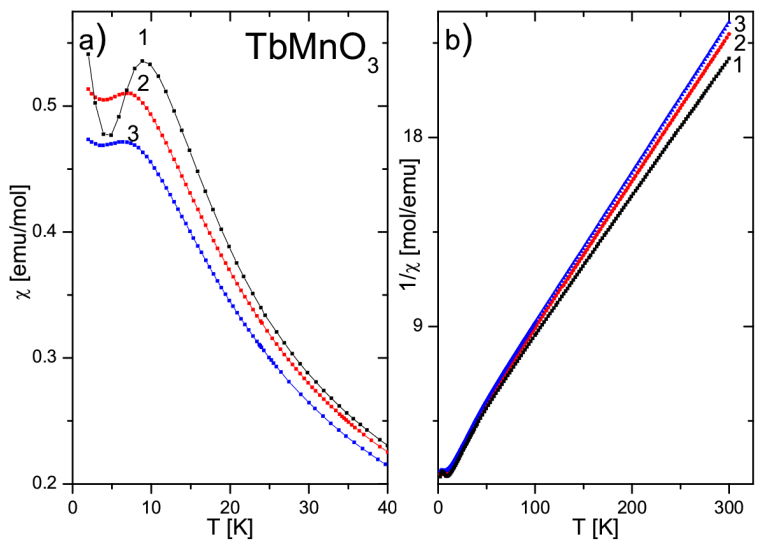

Fig. 3. Temperature dependence of (a) magnetic susceptibility at low temperatures and (b) reciprocal magnetic susceptibility for the polycrystalline (1) and nanoparticles (2 and 3) samples from 800 and $850^{\circ} \mathrm{C}$.

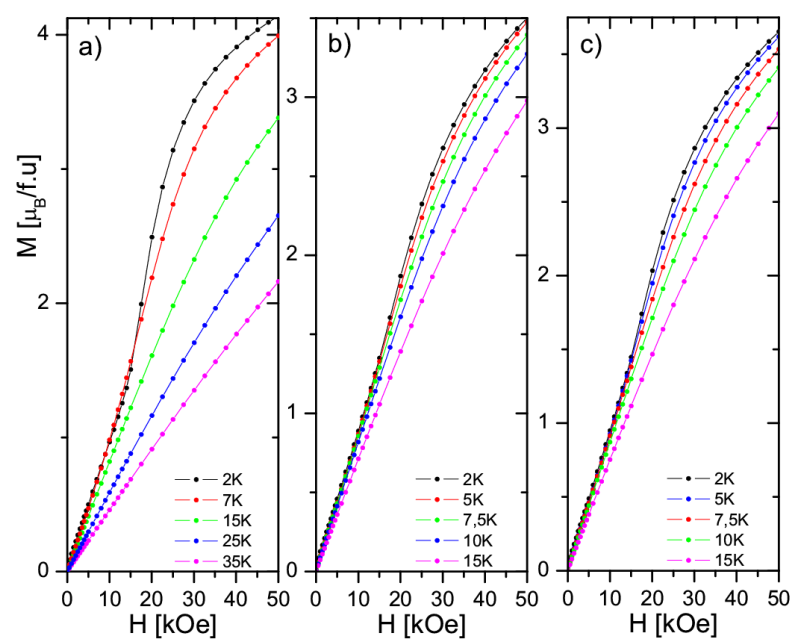

Fig. 4. Magnetization isotherms for the polycrystalline (a) and nanoparticle (b and c) samples from 800 and $850{ }^{\circ} \mathrm{C}$ measured at different temperatures.

decreases with decreasing sintering temperature. The magnetic moments per formula at $2 \mathrm{~K}$ in magnetic field of $50 \mathrm{kOe}$ are equal to $4.14 \mu_{\mathrm{B}}$ for polycrystalline sample, $3.65 \mu_{\mathrm{B}}$ and $3.50 \mu_{\mathrm{B}}$ for nano $850^{\circ} \mathrm{C}$ and nano $800^{\circ} \mathrm{C}$, respectively.

\subsection{Magnetic structure}

In $\mathrm{TbMnO}_{3}$ (space group Pbnm) the $\mathrm{Mn}^{3+}$ ions in the $4 \mathrm{~b}$ site can be described by four Bravais lattices labeled as: $\operatorname{Mn}_{1}(1 / 2,0,0) ; \operatorname{Mn}_{2}(1 / 2,0,1 / 2) ; \operatorname{Mn}_{3}(0,1 / 2,1 / 2)$; $\mathrm{Mn}_{4}(0,1 / 2,0)$. Therefore, four modes are possible, namely, one ferromagnetic arrangement: $\boldsymbol{F}=m_{1}+$ $m_{2}+m_{3}+m_{4}$ and three antiferromagnetic arrangements: $\boldsymbol{A}=m_{1}-m_{2}-m_{3}+m_{4}, \boldsymbol{C}=m_{1}+m_{2}-m_{3}-m_{4}$ and $\boldsymbol{G}=m_{1}-m_{2}+m_{3}-m_{4}$ can be distinguished [11].

The Tb atoms with the magnetic moments occupy the following position in crystal unit cell: $\operatorname{Tb}_{5}(x, y, 1 / 4)$,
$\operatorname{Tb}_{6}(\bar{x}, \bar{y}, 3 / 4), \operatorname{Tb}_{7}(1 / 2+x, 1 / 2-y, 3 / 4)$ and $\operatorname{Tb}_{8}(1 / 2-$ $x, 1 / 2+y, 1 / 4)$. Similarly to the Mn sublattice, four magnetic modes can be defined: $\boldsymbol{F}=m_{5}+m_{6}+m_{7}+m_{8}$ and three antiferromagnetic arrangements: $\boldsymbol{A}=m_{5}$ $m_{6}-m_{7}+m_{8}, \boldsymbol{C}=m_{5}+m_{6}-m_{7}-m_{8}$, and $\boldsymbol{G}=$ $m_{5}-m_{6}+m_{7}-m_{8}[11]$.

\subsubsection{Microsized reference sample}

Figure 5a shows the parts of the neutron diffraction patterns containing the magnetic reflections. For polycrystalline sample at $41 \mathrm{~K}$ and below the additional peaks for $2 \theta$ equal to $19.7^{\circ}, 36.4^{\circ}$ and $45.0^{\circ}$ are observed. These peaks are indexed as the reflections $001^{ \pm}, 111^{-}$and $111^{+}$ connected with the propagation vector $\boldsymbol{k}=\left(0, k_{y}, 0\right)$, where $k_{y}$ is equal to $0.296(3)$ at $41 \mathrm{~K}$ and decreases down to $0.280(2)$ at $5 \mathrm{~K}$. Magnetic order in the Mn sublattice is collinear of $A_{y}$-type in the temperature range $21-41 \mathrm{~K}$. The Mn moments are parallel to the $b$-axis (Fig. 6a). Temperature dependence of the intensity of $001^{ \pm}$peaks gives the Nèel temperature equal to $43 \mathrm{~K}$. At $16 \mathrm{~K}$, the additional $011^{-}$peak at $2 \theta=25.2^{\circ}$ connected with $G$-type is observed. This is due to rise of a noncollinear magnetic structure described by $A_{y} G_{z}$-mode with the Mn moment deviated from $b$-axis (Fig. $6 \mathrm{~b}$ ).

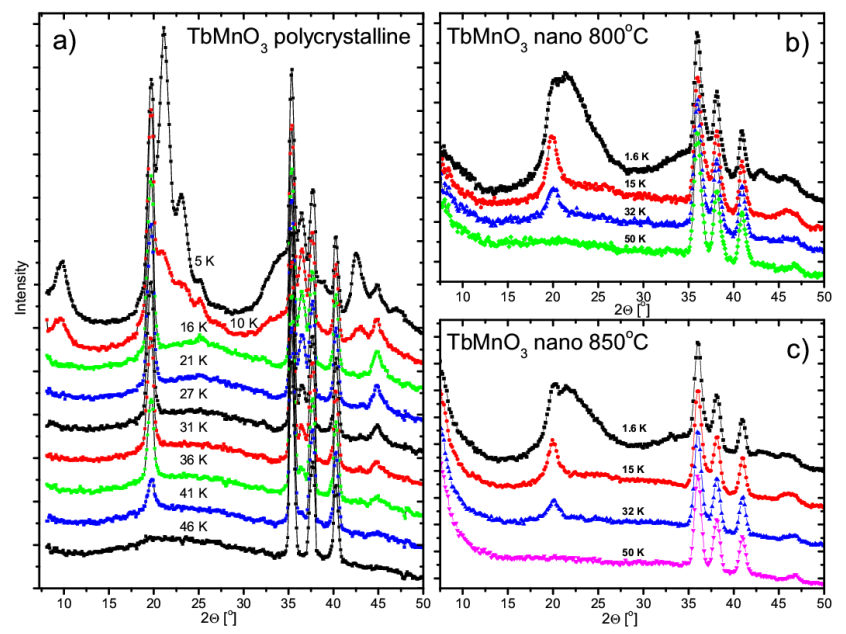

Fig. 5. The low angle neutron diffraction patterns for (a) poly $(\lambda=2.4 \AA)$ and (b) and (c) for nanosamples $(\lambda=2.447 \AA)$ of $\mathrm{TbMnO}_{3}$ measured at different temperatures.

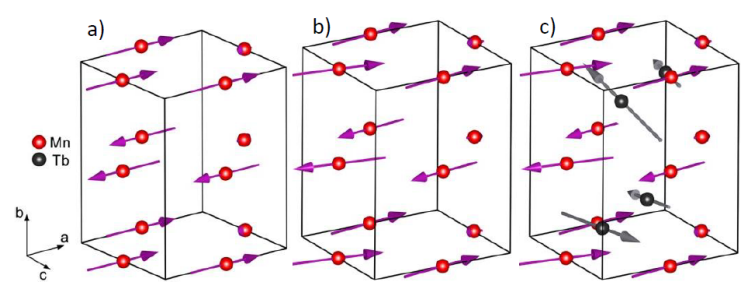

Fig. 6. Magnetic structure of polycrystalline $\mathrm{TbMnO}_{3}$ for Mn-sublattice at (a) $31 \mathrm{~K}$, (b) at $16 \mathrm{~K}$ and (c) for $\mathrm{Mn}$ and $\mathrm{Tb}$ sublattice at low temperatures. 
At $5 \mathrm{~K}$ in the neutron diffraction patterns a number of additional peaks at $2 \theta=9.9^{\circ}, 21.1^{\circ}, 23.0^{\circ}, 42.5^{\circ}$, $47.1^{\circ}$ and broad peak near $35.0^{\circ}$ appear. These peaks are indexed by the propagation vector $\boldsymbol{k}=\left(0, k_{y}, 0\right)$ with $k_{y}=0.432(1)$ as $000^{ \pm}, 001^{ \pm}, 011^{-}, 011^{+}, 111^{-}, 101^{ \pm}$, $021^{-}$and $102^{ \pm}$. These peaks correspond to the different modes: $000^{ \pm}-F, 001^{ \pm}, 021^{ \pm}$and $111^{-}-A$ and $011^{-}$ and $021^{-}-G$. The numerical analysis of intensities gives the magnetic structure described by the $G_{x} A_{y} F_{z^{-}}$ -modes (see Fig. 6c) with the following components of the magnetic moment: $\mu_{x}=6.47(3) \mu_{\mathrm{B}}, \mu_{y}=1.50(8) \mu_{\mathrm{B}}$ and $\mu_{z}=1.0(1) \mu_{\mathrm{B}}$ and a total value of $6.7(1) \mu_{\mathrm{B}}$. The reliability factors are equal to $4.7 \%$ for the $\mathrm{Mn}$ sublattice and $7.07 \%$ for the $\mathrm{Tb}$ sublattice.

It should be mentioned that the pattern measured at $10 \mathrm{~K}$ exhibits some diffused-like scattering originating from the $\mathrm{Tb}$ magnetic moments (at $2 \theta=22^{\circ}-36^{\circ}$ ). This may indicate an existence of short-range correlations of the $\mathrm{Tb}$ magnetic moments. Apart from that, the refined FWHM parameters of the Tb sublattice are about a rank greater than for the Mn sublattice. This suggests that the domain-size related to the $\mathrm{Tb}$ magnetic ordering is significantly smaller than in case of the Mn sublattice. The $\mathrm{Tb}$ moments form the cluster structure. This is in good agreement with the results presented in Ref. [1].

\subsubsection{Nanosized samples}

The neutron diffraction patterns for both nanosamples are shown in Figs. 2, 5b and 5c. In the patterns collected at 15 and $32 \mathrm{~K}$ the additional peaks at $19.9^{\circ}$ and $45.0^{\circ}$ are observed. The results obtained suggest that at these temperatures the Mn magnetic moments form a noncollinear structure described by $A_{y}$-mode with the propagation vector $\boldsymbol{k}=\left(0, k_{y}, 0\right)$ (Fig. 7a). The determined values of the Mn-moments and the $k_{y}$ component of the propagation vector are listed in Table III.
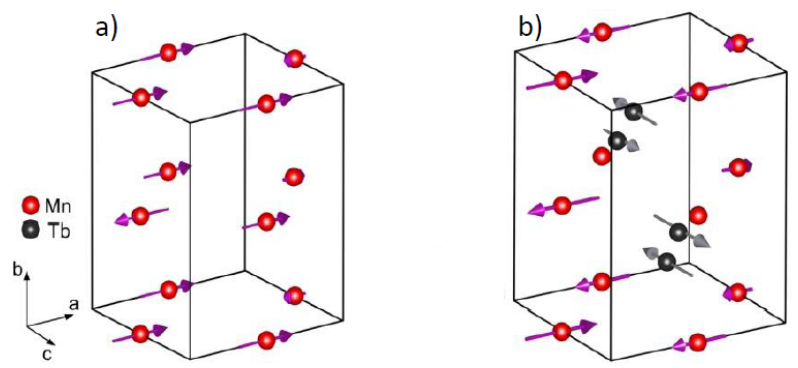

Fig. 7. Magnetic structure of nano-800 specimen: (a) Mn sublattice at $15 \mathrm{~K}$ and (b) $\mathrm{Mn}$ and $\mathrm{Tb}$ sublattice at $1.6 \mathrm{~K}$.

TABLE III

Parameters of the magnetic structure for nano 800 and nano 850 samples of $\mathrm{TbMnO}_{3}$.

\begin{tabular}{c|c|c|c|c|c|c}
\hline \hline \multirow{2}{*}{$T[\mathrm{~K}]$} & \multicolumn{3}{|c|}{ Nano-800 } & \multicolumn{3}{c}{ Nano-850 } \\
\cline { 2 - 7 } & $\mu_{\mathrm{Mn}}\left[\mu_{\mathrm{B}}\right]$ & $k_{y}$ & $R_{\mathrm{m}}[\%]$ & $\mu_{\mathrm{Mn}}\left[\mu_{\mathrm{B}}\right]$ & $k_{y}$ & $R_{\mathrm{m}}[\%]$ \\
\hline 32 & $1.60(12)$ & $0.326(4)$ & 19.2 & $1.35(15)$ & $0.326(5)$ & 20.9 \\
15 & $2.29(8)$ & $0.312(2)$ & 11.5 & $2.08(10)$ & $0.315(2)$ & 19.1 \\
1.6 & $2.94(2)$ & $0.321(2)$ & 10.9 & $3.03(4)$ & $0.328(2)$ & 13.1 \\
$\mu_{\mathrm{Tb}}^{*}\left(\mu_{\mathrm{B}}\right)$ & $3.68(11)$ & $0.443(5)$ & 9.2 & $4.43(7)$ & $0.451(3)$ & 9.3 \\
\hline
\end{tabular}

* data from $1.6 \mathrm{~K}$

At $1.6 \mathrm{~K}$, the additional broad maxima at $2 \theta \approx 22^{\circ}$, $33^{\circ}$ and $43^{\circ}$ are observed. These maxima contain information on the magnetic ordering in the $\mathrm{Tb}$ sublattice. Absence of the $000^{ \pm}$peaks at $2 \theta \approx 10^{\circ}$ indicates the absence of the ferro- $F$ component. The performed numerical analysis reveals a noncollinear magnetic structure of Tb moments described by $G_{x} A_{y}$ mode and propagation vector $\boldsymbol{k}=\left(0, k_{y}, 0\right)$ (Fig. $\left.7 \mathrm{~b}\right)$. The obtained values of the $\mathrm{Tb}$ magnetic moment and $k_{y}$ component are listed in Table III. The calculation was performed on assumption of the different parameters $U, V, W$ for $\mathrm{Tb}$ magnetic reflection than for other peaks (nuclear and Mn magnetic).

Apart from lack of the $G$ (Mn sublattice) and $F(\mathrm{~Tb}$ sublattice) modes, the $k_{y}$ components evidenced for the nanospecimens are larger than in the conventional polycrystalline sample (both for $\mathrm{Tb}$ and Mn sublattices).

\section{Discussion}

In the paper, comparison of the magnetic properties, crystal and magnetic structures in poly- and nanosamples of $\mathrm{TbMnO}_{3}$ is presented. X-ray and neutron diffraction data indicate that all the samples have the orthorhombic crystal structure described by Pbnm space group with the small difference of the structural parameters, which influence the Mn-distance in the $a-b$ plane.

Temperature dependence of the magnetic susceptibility for nanosamples shows the broad maximum at low temperatures (Fig. 3), which according to the neutron diffraction data is connected with the magnetic ordering of the $\mathrm{Tb}$ moments. Comparison of the data for nanoand poly-samples suggests that the ordering in the $\mathrm{Tb}$ sublattice is similar to the cluster type ordering. The 
critical temperature for the Mn sublattice was not determined from the magnetic data because the large $\mathrm{Tb}$ moment strongly influences the temperature dependence of the magnetic susceptibility. Only some deviations from the Curie-Weiss law, observed below $50 \mathrm{~K}$ for all the samples are a manifestation of ordering of the magnetic moment in the Mn sublattice. Above this temperature, the reciprocal magnetic susceptibility obeys the CurieWeiss law with the negative value of the paramagnetic Curie temperature indicating antiferromagnetic interactions. The effective magnetic moment is equal to the sum of the magnetic moments of the free $\mathrm{Tb}^{3+}$ and $\mathrm{Mn}^{3+}$ ions for polycrystalline and is smaller for nanosamples. Neutron diffraction data confirmed magnetic ordering of the $\mathrm{Mn}$ sublattice in all investigated specimens below $43 \mathrm{~K}$, however some significant differences in the observed structures were noticed. Namely, for the reference sample Mn sublattice exhibits two magnetic phases: in between 43-16 K ( $A$-mode) and below $16 \mathrm{~K}$ ( $G A$-mode), while for nanospecimens only $A$-mode was recognized. For $\mathrm{Tb}$ sublattice a lack of the ferromagnetic component in case of nanospecimens must be underlined. Moreover, reflections related to the $\mathrm{Tb}$ sublattice in both nanosamples are significantly broader than for the reference hinting at cluster-like ordering.

For nanosamples, the observed decrease of the effective magnetic moments seems to be connected to rise of the $\mathrm{Mn}^{4+}$ concentration [10]. On the other hand, lowering of the saturation magnetic moments under external magnetic fields can be explained by spin disorder in a surface shell of nanogranular systems as proposed in Ref. [12].

The interactions between Mn moments based on the exchange integrals were discussed by Bertaut [11] and applied to the case of $\mathrm{o}-\mathrm{YMnO}_{3}$ [13]. According to the authors of Ref. [13] the eigenvalue corresponding to the $A$ mode, along the direction $\boldsymbol{k}=k_{y} \boldsymbol{b}^{*}$, can be written as

$$
\begin{aligned}
& \lambda_{A}\left(0, k_{y}, 0\right)=2\left(J_{11 a}-J_{12}\right)+2 J_{11 b} \cos \left(2 \pi k_{y}\right) \\
& \quad-4\left(2 J_{13}-J_{14}\right) \cos \left(\pi k_{y}\right) .
\end{aligned}
$$

The derivative of $\lambda_{A}$ with respect to $k_{y}$ :

$$
\begin{aligned}
& \partial \lambda_{A} / \partial k_{y}=4 \pi \sin \left(\pi k_{y}\right) \\
& \quad \times\left[-2 J_{11 b} \cos \left(\pi k_{y}\right)+2 J_{13}-J_{14}\right]=0 .
\end{aligned}
$$

This condition is fulfilled only when $k_{y}=0$ (simple antiferromagnetic mode) or $\cos \left(\pi k_{y}\right)=\left(2 J_{13}-J_{14}\right) / 2 J_{11 b}$ (modulated structure). For $\left(2 J_{13}-J_{14}\right)<0$ the $A$ mode is energetically preferable. According to Ref. [14] increase of $k_{y}$ means that $\left(2 J_{13}-J_{14}\right) / 2 J_{11 b}$ decreases. This could be due to decreasing $J_{14}$ caused by a change of $\mathrm{Mn}_{1}-\mathrm{O}_{2}-\mathrm{Mn}_{4}$ angle or increase of the $J_{11 b}$ exchange integral by super-superexchange mechanism along the $b$-axis. The data in Table I suggest that the decrease of the $b$-axis, evidenced for nanosamples, is an origin of increase of $J_{11 b}$ exchange integral. Moreover, a small decrease of the $\mathrm{Mn}_{1}-\mathrm{O}_{2}-\mathrm{Mn}_{4}$ angle is observed in nanosamples leading to a lowering of $J_{14}$ (see Table II).

The inelastic neutron scattering for $\mathrm{TbMnO}_{3}$ yields the positive value of the exchange interaction in the basal plane $\left(J_{14} \approx 0.15(1) \mathrm{meV}\right)$ and negative value $\left(J_{13} \approx-0.31(2) \mathrm{meV}\right)$ along $c$-axis [15]. It means that $\left(2 J_{13}-J_{14}\right)$ is negative in case of $\mathrm{TbMnO}_{3}$. Therefore, the $A$-mode, observed for all specimens, was expected. For $k_{y}=0.296$ obtained just below $T_{\mathrm{N}}$, for poly-sample, the condition $\cos \left(\pi k_{y}\right)=\left(2 J_{13}-J_{14}\right) / 2 J_{11 b}$ gives $J_{14} \approx 2\left(J_{13}-0.6 J_{11 b}\right)$ which is positive. The assumption of negative superexchange $J_{13}$ and $J_{11 b}$ interactions raises a condition: $0.6\left|J_{11 b}\right|>\left|J_{13}\right|$. In the case of nanosample $k_{y}$ equal to 0.328 gives $\cos \left(\pi k_{y}\right)=0.51$ and similar condition: $0.51\left|J_{11 b}\right|>\left|J_{13}\right|$.

The utmost importance of surface-to-bulk ratio on concerns magnetic properties of $\mathrm{RMnO}_{3}$ was issued in Refs. [16-18] where thin films of $\mathrm{TbMnO}_{3}$ and $\mathrm{YbMnO}_{3}$ were investigated. The general conclusion was that magnetic properties change dramatically when dimensionality is restricted. For example, in thin films of $\mathrm{TbMnO}_{3}$ purely ferromagnetic behavior was observed [17]. In the case of $\mathrm{YbMnO}_{3}$ a critical layer thickness of $30 \mathrm{~nm}$ was claimed to be a border between bulk and reduced dimensionality magnetic behavior [18].

\section{Acknowledgments}

This research project has been supported by the European Commission under the 6th Framework Programme through the Key Action: Strengthening the European Research Area, Research Infrastructures. Contract no: RII3-CT-2003-505925 (NMI3).

\section{References}

[1] S. Quezel, F. Tcheou, J. Rossat-Mignod, G. Quezel, E. Roudaut, Physica B 86-88, 916 (1977).

[2] R. Kajimoto, H. Yoshizawa, H. Shintani, T. Kimura, Y. Tokura, Phys. Rev. B 70, 012401 (2004).

[3] O. Prokhenenko, R. Feyerherm, M. Mostovoy, N. Aliouane, E. Dudzik, A.U.B. Wolter, A. Maljuk, D.N. Argyrion, Phys. Rev. Lett. 99, 177206 (2007).

[4] M. Kenzelmann, A.B. Haris, S. Jones, C. Broholm, J. Schefer, S.B. Kim, C.L. Zhang, S.-W. Cheong, O.P. Vajk, J.W. Lynn, Phys. Rev. Lett. 95, 087206 (2005).

[5] J. Blasco, C. Ritter, J. Garcia, J.M. de Teresa, J. Pèrez-Cacho, M.R. Ibarra, Phys. Rev. B 62, 5609 (2000).

[6] T. Kimura, T. Goto, H. Shiutani, K. Ishizaka, T. Arima, Y. Tokura, Nature 426, 55 (2003).

[7] T. Kimura, G. Lawet, T. Goto, Y. Tokura, A.P. Ramirez, Phys. Rev. B 71, 224425 (2005).

[8] V. Dyakonov, A. Szytula, R. Szymczak, E. Zubov, A. Szewczyk, Z. Kravchenko, W. Bazela, K. Dyakonov, A. Zarzycki, V. Varyukhin, H. Szymczak, Low Temp. Phys. 38(3), 1 (2012).

[9] J. Rodriguez-Carvajal, Physica B 192, 55 (1993).

[10] T.I. Arbuzova, B.A. Gizhevski, R.G. Zakharov, S.A. Petrova, N.M. Chebotaev, Phys. Solid State 50, 1487 (2008)

[11] E.F. Bertaut, Magnetism, Vol. III, Eds. G.T. Rado, H. Shul, Academic Press, New York 1963, p. 149. 
[12] M.A. Lopez-Quintela, L.E. Hueso, J. Rivas, F. Rivadulla, Nanotechnology 14, 212 (2003).

[13] S. Quezel, J. Rossat-Mignod, E.F. Bertaut, Solid State Commun. 14, 941 (1974).

[14] H.W. Brinks, J. Rodriguez-Carvajal, H. Fjellvåg, A. Kjekshus, B.C. Hauback, Phys. Rev. B 63, 094411 (2001).

[15] D. Senff, P. Link, K. Hradil, A. Hiess, L.P. Regnault, Y. Sidis, N. Aliouane, D.N. Argyriou, M. Braden, Phys. Rev. Lett. 98, 137206 (2007).
[16] E. Galstyan, B. Lorenz, K.S. Martirosyan, F. Yen, Y.Y. Sun, M.M. Gospodinov, C.W. Chu, J. Phys., Condens. Matter 20, 325241 (2008).

[17] B.J. Kirby, D. Kan, A. Luykx, M. Murakami, D. Kundaliya, I. Takeuchi, J. Appl. Phys. 105, 07D917 (2009).

[18] D. Rubi, S. Venkatesan, B.J. Kooi, J.Th.M. De Hosson, T.T.M. Palstra, B. Noheda, Phys. Rev. B 78, 020408(R) (2008). 